\title{
The challenge of diagnosing common dermatomycosis by reflectance confocal microscopy
}

\section{Running title: RCM in diagnosing common dermatomycosis}

\author{
Jinrong Zeng ${ }^{1}$, Hanyi Zhang ${ }^{2}$, Yue Zhang ${ }^{2}$, Lihua Gao ${ }^{1}$, Jian Huang ${ }^{1}$, Zhen Tang ${ }^{1 *}$ and Jianyun Lu ${ }^{{ }^{*}}$ \\ ${ }^{1}$ Department of Dermatology, Third Xiangya Hospital, Central South University, No. 138, Tongzipo Road, Yuelu District, Changsha, Hunan, China, \\ 410013 \\ ${ }^{2}$ Xiang Ya School of Medicine, Central South University, Changsha, 410013, Hunan, China
}

*Corresponding Author: Jianyun Lu and Zhen Tang, The Third Xiangya Hospital, Central South University, No.138 Tongzipo Rd, Yuelu District, Changsha, Hunan, China, 410013.

Received date: August 26, 2021; Accepted date: September 15, 2021; Published date: September 20, 2021

Citation: J Zeng, H Zhang, Y Zhang, Z Tang, J Lu, et al. (2021) The challenge of diagnosing common dermatomycosis by reflectance confocal microscopy. J. Dermatology and Dermatitis. 6(3); DOI: 10.31579/2578-8949/085

Copyright: @2021 Jianyun Lu and Zhen Tang, This is an open-access article distributed under the terms of The Creative Commons. Attribution License, which permits unrestricted use, distribution, and reproduction in any medium, provided the original author and source are credited.

\begin{abstract}
Background: Traditional detection of fungal infections of the skin relies on microscopy techniques or fungal culture. Currently, reflectance confocal microscopy (RCM) has been widely applied to assist the diagnosis of common dermatomycosis with advantages of non-invasiveness, celerity, real time, and repeatability.

Materials and Methods: A total of 478 clinically suspected dermatomycosis patients were enrolled in this study including 148 cases of tinea manus and pedis, 188 cases of tinea corporis and cruris and 142 cases of pityriasis versicolor. RCM examination was performed to image the lesions.

Aim: This study aimed to summarize the image characteristics of in vivo RCM examination on common dermatomycosis and retrospectively evaluate its accuracy as compared with microscopy results. Furthermore, we attempted to tackle the challenges of RCM diagnosis on common dermatomycosis.

Results: Based on RCM images, 231 of 478 (48.3\%) patients were detected with hyphae. Among all RCM confirmed cases, 58 out of $148(39.2 \%)$ were tinea manus and pedis, 145 out of $188(77.1 \%)$ were tinea corporis and cruris, and 28 out of $142(19.7 \%)$ were pityriasis versicolor. The remaining patients $(51.7 \%)$ could not be diagnosed by the dermatologist according to RCM. Hyphae structures were primarily identified during diagnoses of dermatomycosis by RCM.
\end{abstract}

Conclusions: RCM is a novel optical imaging technique that confers high-resolution images of fungi. RCM has certain advantages in the diagnosis of tinea manus and pedis. RCM is not suitable for the diagnosis of pityriasis versicolor.

Keywords: dermatomycosis; reflectance confocal microscopy; tinea manus; pedis; tinea corporis; cruris; pityriasis versicolor

\section{Introduction}

Dermatomycosis are infectious skin diseases caused by superficial fungi. According to the depth and location of tissues that fungi invade, they can be divided into superficial mycosis, deep mycosis and systemic mycosis. Among them, common types of dermatomycosis such as tinea manus, tinea pedis, tinea corporis, tinea cruris and pityriasis versicolor affect $20 \%$ to $25 \%$ of population in the world and the incidence has increased continually in recent years $[1,2]$. Superficial mycosis mainly invades the skin mucous membranes and their appendages. Dermatophytes such as trichophyton, microsporum and epidermophyton are the main pathogens causing superficial mycosis; they grow in the form of hyphae or spores [3]. They all invade superficial keratinized tissues (skin, hair, fingernails and toenails) instead of deep tissues or internal organs, which are difficult to be identified and their cultivation requirements are very demanding $[4$, 5]. At present, the diagnostic basis for superficial mycosis is that the patient has a typical clinical manifestation with a positive result of fungal microscopy and/or cultural isolation of dermatophytes $[6,7]$. 
Reflectance confocal microscopy (RCM) is a new technique to achieve three-dimensional imaging of skin structures using the refractive index differences of various cellular constituents such as melanin and keratin $[8,9]$. Many studies have reported that RCM was not only applied to diagnose skin mycosis but also nail and hair dermatophytosis [10, 11]. Moreover, due to its advantages of painless, real time and convenience, [12] it has been used to detect inflammatory skin diseases, pigmented skin diseases, infectious skin diseases and skin tumors [13-15]. At present, it is not well known whether it can be applied to diagnose dermatomycosis independently and what is the potential diagnostic accuracy on dermatomycosis [16].

In this study, we retrospectively analyzed 478 patients with clinically suspected dermatomycosis; all of them received RCM examinations. We summarized their image characteristics. We evaluated the clinical applicability and accuracy of RCM in the diagnosis of common dermatomycosis as compared with fungal fluorescence microscopy results.

\section{Material and Methods}

\section{Patients}

We retrospectively analyzed 478 patients (females, $n=183$; males, $n=295$ ) ranging from 3 to 76 years of age (mean= 38.4 years old) who were clinically probable diagnosis of tinea manus, tinea pedis, tinea cruris, tinea corporis, or pityriasis versicolor between January 2017 and December 2019 in the Department of Dermatology, Third Xiangya Hospital of Central South University. All patients underwent RCM examinations and 146 of 478 cases were performed a fungal microscopy test. This study was approved by the Ethics Committee of the Third Xiangya Hospital.

\section{RCM imaging}

All patients were examined by a professional dermatologist (an attending physician with more than five years of professional training) using the Vivascope 1500 RCM device (Lucid Inc, Rochester, NY, USA) [17]. Patients took appropriate postures and fully exposed skin lesions area in resting state, the dermatologist selected the fresh skin lesion with a few scales as inspection area. The dermatologist adjusted the scan head to make it on flat lesions when imaging, scanning power varied from $0 \mathrm{~mW}$ to $16 \mathrm{~mW}$, scanning area was $500 \mu \mathrm{m} * 500 \mu \mathrm{m}$, horizontal movement range was $0-8 \mathrm{~mm}$. The wavelength of the light source was $830 \mathrm{~nm}$. Prior to each test, the objective lens was infiltrated with pure water, and the water between the objective lens and the window was optically corrected.
More light could help penetrate the epidermis and superficial dermis to obtain high-quality images. At last, the dermatologist identified and saved the best images [18]. The clinician made a diagnosis based on RCM images. When the RCM image quality was poor or did not show a reliable diagnosis basis, the case was judged to be undiagnosable.

\section{Fungal fluorescence microscopy}

146 patients underwent a skin fungal microscopy examination in parallel. The skin lesions were disinfected with $75 \%$ alcohol, a small amount of dander was scraped and placed on the glass slide, then we added a drop of fluorescent staining solution (Litts fungal fluorescent staining solution type I, 200 servings per box, Jiangsu NuoGe Biotechnology Co. LTD, China), covered with a cover slide, stained for $1 \mathrm{~min}$, observed under a fluorescent microscope, recorded and photographed. Hyphae or spore structures found under the microscope were considered positive, otherwise negative [19, 20].

\section{Statistical analysis}

Comprehensive and descriptive analysis of RCM results and fungal fluorescence microscopy results were performed using SPSS 23.0 (SPSS Inc, Chicago, USA). All of the diagrams and graphs reporting cumulative data were performed using GraphPad Prism 6.0 (Graphpad Software Inc, California, USA).

\section{Results}

\section{Characteristics of RCM images}

The features of RCM images from patients with tinea manus and pedis were high refractive index and branched elongated hyphae in the middle and lower layers of the stratum corneum. In addition, pustule structures surrounded by a large number of inflammatory cells could be observed in those profound inflammatory areas; RCM images from patients with tinea corporis and cruris showed separated hyphae in the middle of the stratum corneum; some cases showed round low-index blisters and inflammatory cells aggregation in the stratum corneum and spinous layer, dermal papilla. Hyperemia and expansion of superficial blood vessels were observed and surrounded by a few inflammatory cells. The hyphae in the groin areas appeared as continuous elongated and high-refractive structures; most of the hyphae in the trunk area appeared as short and thick structures. RCM images from patients with pityriasis versicolor showed medium-refractive short thick hyphae in the upper stratum corneum, mild edema of acanthocytes, and infiltration of sparse inflammatory cells around the dermal papilla and superficial vessels (Figure1). 

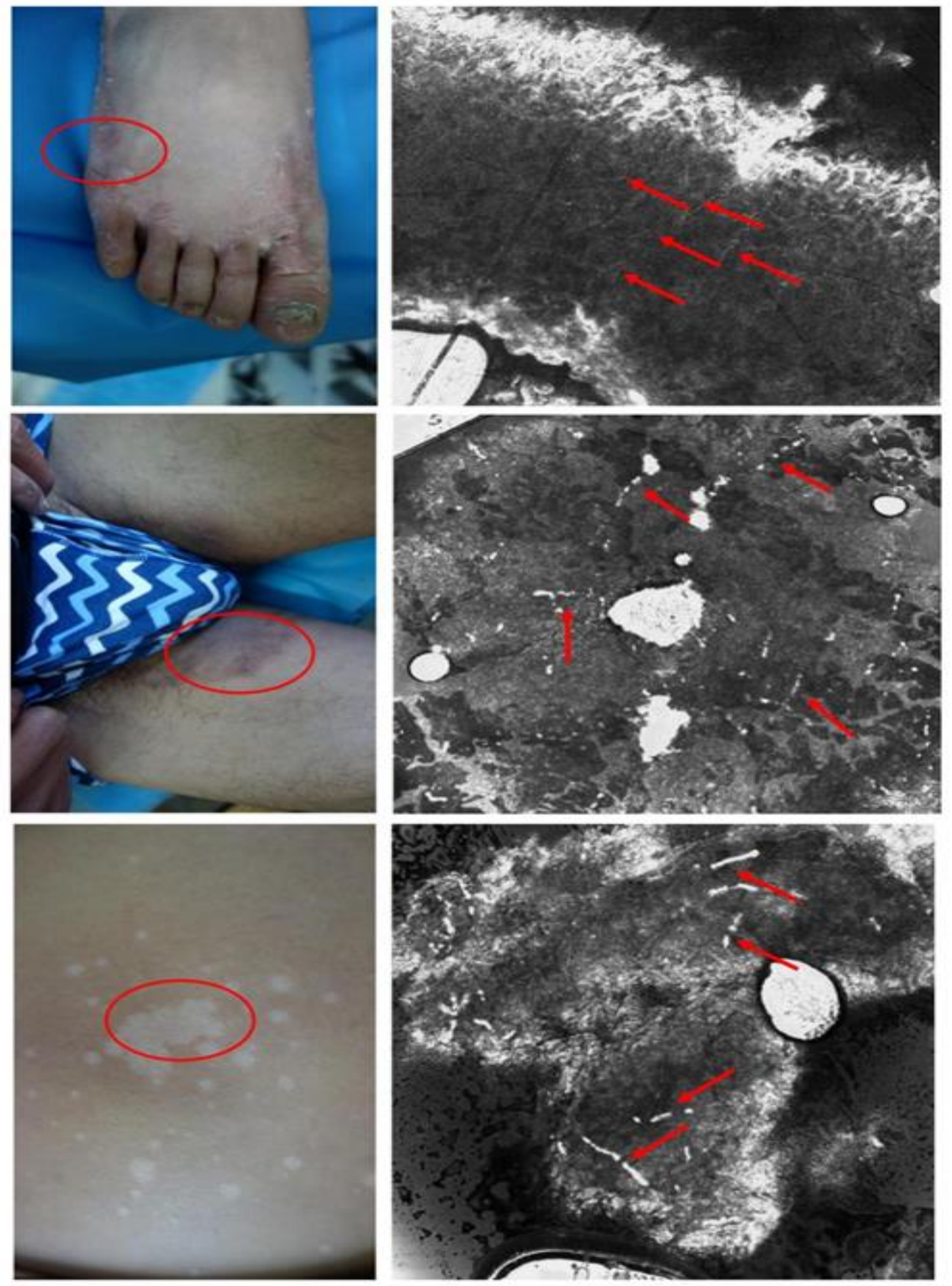

Figure 1: The typical characteristics of RCM images 


\section{Diagnosis by RCM}

Based on the reports from RCM images, we summarized the diagnostic characteristics and positive rate of RCM in 478 cases in Table1. Furtherly, we analyzed the number of undiagnosable cases for RCM images and the body sites of imaging in Table 2 .

\begin{tabular}{|c|c|c|c|c|c|c|c|}
\hline \multirow[t]{2}{*}{ Dermatomycoses } & \multirow{2}{*}{$\begin{array}{c}\text { Cases } \\
\text { number }\end{array}$} & \multicolumn{3}{|c|}{ Microscopy(+) features } & \multirow{2}{*}{$\begin{array}{c}\text { RCM } \\
\text { diagnosis } \\
(+)\end{array}$} & \multirow{2}{*}{$\begin{array}{c}\text { RCM } \\
\text { diagnosis (-) }\end{array}$} & \multirow[t]{2}{*}{ Positive rate } \\
\hline & & $\begin{array}{l}\text { A large } \\
\text { number of } \\
\text { hyphae }\end{array}$ & \begin{tabular}{l}
\multicolumn{1}{c}{ A } \\
moderate \\
number of \\
hyphae
\end{tabular} & $\begin{array}{l}\text { A small } \\
\text { number of } \\
\text { hyphae }\end{array}$ & & & \\
\hline Tinea manus and pedis & 148 & 10 & 5 & 43 & 58 & 90 & $39.2 \%$ \\
\hline Tinea corporis and cruris & 188 & 29 & 19 & 97 & 145 & 43 & $77.1 \%$ \\
\hline pityriasis versicolor & 142 & 2 & 3 & 23 & 28 & 114 & $19.7 \%$ \\
\hline
\end{tabular}

Table 1: The diagnostic characteristics and positive rate of RCM in 478 cases

Notes (A large number of hyphae: the number of hyphae is greater than or equal to 5; A medium number of hyphae: the number of hyphae is greater than or equal to 3 and less than 5; A small number of hyphae: the number of hyphae is greater than or equal to 1 and less than 3); (+) represents a positive result and (-) represents a negative result.

\begin{tabular}{|l|c|c|c|c|c|}
\hline Dermatomycoses & $\begin{array}{c}\text { Face } \\
\text { and neck }\end{array}$ & $\begin{array}{c}\text { Tr } \\
\text { unk }\end{array}$ & $\begin{array}{c}\text { Extrim } \\
\text { iti s }\end{array}$ & $\begin{array}{c}\text { Groin, perineal } \\
\text { and perianal areas }\end{array}$ & $\begin{array}{c}\text { Palms } \\
\text { and soles }\end{array}$ \\
\hline Tinea manus and pedis(n=90) & 0 & 0 & 0 & 0 & 90 \\
\hline Tinea corporis and cruris(n=43) & 8 & 17 & 7 & 11 & 0 \\
\hline pityriasis versicolor(n=114) & 41 & 66 & 7 & 0 & 0 \\
\hline Total(n=247) & 49 & 83 & 12 & 11 & 92 \\
\hline
\end{tabular}

Table 2: The undiagnosable body sites statistics of RCM imaging

\section{Comparison of fungal fluorescence microscopy with related $\mathrm{RCM}$ diagnosis}

A total of 146 patients with a RCM examination were performed fungal fluorescence microscopy test in parallel, the comparison analysis results of the two examinations based on 146 cases were showed in Table 3 . We found 95 cases $(65.1 \%)$ were diagnosed as positive, and RCM examination found 65 cases $(44.5 \%)$ were positive.

\begin{tabular}{|l|l|l|}
\hline Dermatomycoses & Microscopy $(+)$ & RCM $(+)$ \\
\hline Tinea manus and pedis $(\mathrm{n}=80)$ & 42 & 34 \\
\hline Tinea corporis and cruris(n=42) & 31 & 27 \\
\hline pityriasis versicolor $(\mathrm{n}=24)$ & 22 & 4 \\
\hline Total $(\mathrm{n}=146)$ & 95 & 65 \\
\hline
\end{tabular}

Table 3: The comparison analysis of the two examinations based on 146 cases

Among 95 cases with positive results of fungal fluorescence microscopy, there were 25 cases of tinea manus and pedis, 26 cases of tinea corporis and cruris, and 4 cases of pityriasis versicolor that were positive under RCM images. Therefore, the RCM false-negative rates were $10.0 \%$ in tinea manus and pedis, $9.5 \%$ in tinea corporis and cruris, and $75.0 \%$ in pityriasis versicolor. Among 51 cases with negative results of fungal microscopy, there were 9 cases of tinea manus and pedis, and 1 case of tinea corporis and cruris that was diagnosed as positive by RCM. The positive rates of tinea manus and pedis after combining fungal microscopy with RCM examinations increased from $52.5 \%$ to $63.8 \%$, tinea corporis and cruris increased from $73.8 \%$ to $76.2 \%$, and the combination of the two tests results of pityriasis versicolor did not increase its diagnostic positive rate. Therefore, RCM could be recommended for those highly suspected patients of tinea manus and pedis with a negative result of fungal fluorescence microscopy; while for patients of probable pityriasis versicolor with a negative result of fungal fluorescence microscopy, a further RCM examination less likely could increase the positive rate (Table4 and Figure2).

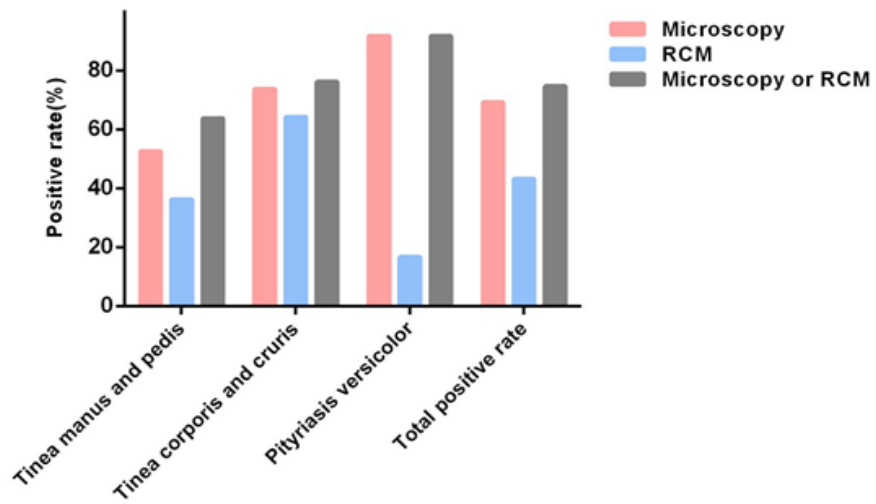




\begin{tabular}{|l|c|c|c|}
\hline Positive rate & Microsc & RCM & Microscopy or RCM \\
\hline Tinea manus and pedis & $52.5 \%$ & $36.3 \%$ & $63.8 \%$ \\
\hline Tinea corporis and cruris & $73.8 \%$ & $64.3 \%$ & $76.2 \%$ \\
\hline pityriasis versicolor & $91.7 \%$ & $16.7 \%$ & $91.7 \%$ \\
\hline Total positive rate & $69.2 \%$ & $43.2 \%$ & $74.7 \%$ \\
\hline
\end{tabular}

Table 4: The positive rate comparison of the two examinations based on 146 cases.

\section{Discussion}

Superficial mycosis is mainly caused by dermatophytes and Malassezia invading keratinized tissues (corneum, hair and nails). The traditional detection method is scraping the lesion site to obtain skin scale tissue for light microscopic observation or fungal culture to determine the pathogen or to confirm the diagnosis by biopsy. At present, domestic and foreign experts have made many progresses in exploring non-invasive skin imaging technology [21].

RCM is a novel optical imaging technique that presents high-resolution images of the skin and relies on a low-power laser emitting near-infrared light $(830 \mathrm{~nm})$ to image the epidermis and papillary layers of the skin [22]. In this study, we used VivaScope1500 and not the hand-held 3000 device which was more suitable for the diagnosis of superficial mycosis due to the possibility of applying the camera on a convex surface and of exploring large skin areas in less time [23]. It has been found that RCM can be used in infectious skin diseases, such as herpes virus infection, human papilloma virus infection and fungal infections [24, 25]. Ramsey Markus et al. [26]first discovered and reported the RCM features of dermatomycosis; Xu Ai'e et al [27]. assessed the value of RCM in dermatophytosis, with a sensitivity of $63.64 \%$ for tinea manus and pedis and $82.61 \%$ for tinea cruris. However, we found that the positive detection rate of RCM in the diagnosis of dermatomycosis was quite different from that of fungal fluorescence microscopy through data analysis, so we explored some limitations faced by the diagnosis of dermatomycosis with RCM in this study.

In this study, all of included cases underwent RCM and 146 of them also performed fungal fluorescence microscopy examination concurrently, we did not perform conventional optical microscopy examination and fungal culture, thus, the overall positive rate of fungal examination was only $69.2 \%$. As a comparison, the overall positive rate of RCM (43.2\%) was obviously different from that of fungal examination $(69.2 \%)$ with the detection of hyphae structures or spores as the diagnostic criteria and there was a large difference in the positive rate of dermatomycosis at different sites.

In this article, we summarized the RCM characteristics and distribution of tinea manus and pedis, tinea corporis and cruris and pityriasis versicolor. Although we could see different manifestations of hyphae, RCM has several limitations in the classification of pathogenic genera. Therefore, it cannot replace fungal culture. At the same time, we found a large number of hyphae structures in the RCM from some patients with tinea manus and pedis, tinea corporis and cruris, and pityriasis versicolor. The inflammatory response in the spinous layer and inflammatory cells infiltration in the dermis were more pronounced in these patients. However, for patients with moderate or small quantity of hyphae structures in the RCM the acanthotic inflammatory response and intradermal inflammatory cell infiltration were not profound, which could be related to the patient's course and the affected skin site. The skin environment of the foot and groin was moist and more suitable for fungal growth and reproduction, so more hyphae could be seen and the inflammatory response was more severe. The positive correlation between the number of hyphae and the severity of inflammation still needed further in-depth study.
In this study, we selected 146 patients for fungal fluorescence microscopy at the same time. Taking the positive microscopy as comparison, RCM shows a certain diagnostic value in the diagnosis of dermatomycosis, thus providing a scientific basis for the diagnosis and treatment of clinicians. However, many patients with positive microscopy had negative RCM results, one possible reason is that the palmar and plantar parts are thick due to friction and the RCM mostly appeared uniform high-refractive image, leading to a neglect of hyphae. As for scaly keratotic tinea manus and pedis, the skin lesions present diffuse roughness, thickening, scaling [28]. Whether the scales or thickened stratum corneum can cause difficulties in diagnosis is yet to be determined. For vesicular tinea manus and pedis, low refractive index blisters can be detected in the stratum corneum and spinous layer, [18] For blister tinea manus and pedis, low refractive index blisters can be detected in the stratum corneum and spinous layer; however, due to the severe inflammatory responses, the erosion or exudation of the lesion may make it difficult for RCM to find hyphae. The positive rate of RCM diagnosis of tinea manus is much lower as compared with tinea pedis, which may be related to the fact that hands often contact with many things and are cleaned frequently. There are many impurities affecting the hyphae resolution or imaging quality. Fungal infections that occur on the trunk area are more likely to cause secondary reactions such as scratches and scabs, which makes it hard to find hyphae structures under the RCM microscope. Since the pathogenic fungus of pityriasis versicolor are usually located in the superficial layer of the stratum corneum, the medium dripped during RCM imaging may remove the dander above the skin as well as the hyphae. Moreover, the hyphae of Malassezia are mostly short, thick and with blunt ends; such morphological characteristics may be difficult to find in the image. In addition, the typical spore structure cannot be identified by the RCM microscope, which may be one of the significant reasons for missed diagnosis. Admittedly, some patients $(n=10)$ with negative microscopy results presented positive RCM results. Most of those pathogens are parasitic in the middle and lower layers of the skin stratum corneum which is easily neglected by fungal microscope due to sampling inefficiency. RCM, however, can scan to the entire epidermis or even the upper and middle layers of the dermis to facilitate the search for pathogens. We have found that different parts and types of superficial mycosis have different parasitic depths. For example, pityriasis versicolor is located in 1 2 layers of RCM with depths of 3.05 6.10 $\mu \mathrm{m}$; tinea manus and pedis is 20 30 layers with depths of 61.00 91.50 $\mu \mathrm{m}$; tinea corporis and cruris is $6 \sim 12$ layers with depths of 18.30 36.60 $\mu \mathrm{m}$. Therefore, RCM examination can be further conducted to screen those highly suspected tinea manus and pedis that are with negative results of fungal microscopy.

Our study has shown that combination of RCM and fungal fluorescence microscopy can significantly increase the positive rates of pathogenic fungi. However, RCM is not suitable for the diagnosis of pityriasis versicolor. RCM still faces many challenges in the diagnosis of dermatomycosis. First, the stratum corneum shows an image with a large brightness area as it is rich in keratin which presents high refractive index, and the refractive index of the hyphae is also high; As a result, hyphae also shows a bright linear shadow in the image. Therefore, it may be difficult to accurately find the hyphae in the stratum corneum area due to poor contrast of image. Accumulation of diagnostic experience is required. Second, the morphology and refractive index of different types of fungal hyphae may be different, which also leads to differences in the 
diagnostic positive rates of different dermatomycosis. Third, most of the superficial fungal disease lesions show squama formation and thickening of the stratum corneum, which can also have high refractive RCM performance. These components of the lesions may also affect the identification of hyphae. The types of fungus also have impacts on imaging. Forth, there are limitations for morphology identifications by RCM which requires experienced physicians to overcome. For example, the RCM images of hyphae manifest linear intermittent or continuous
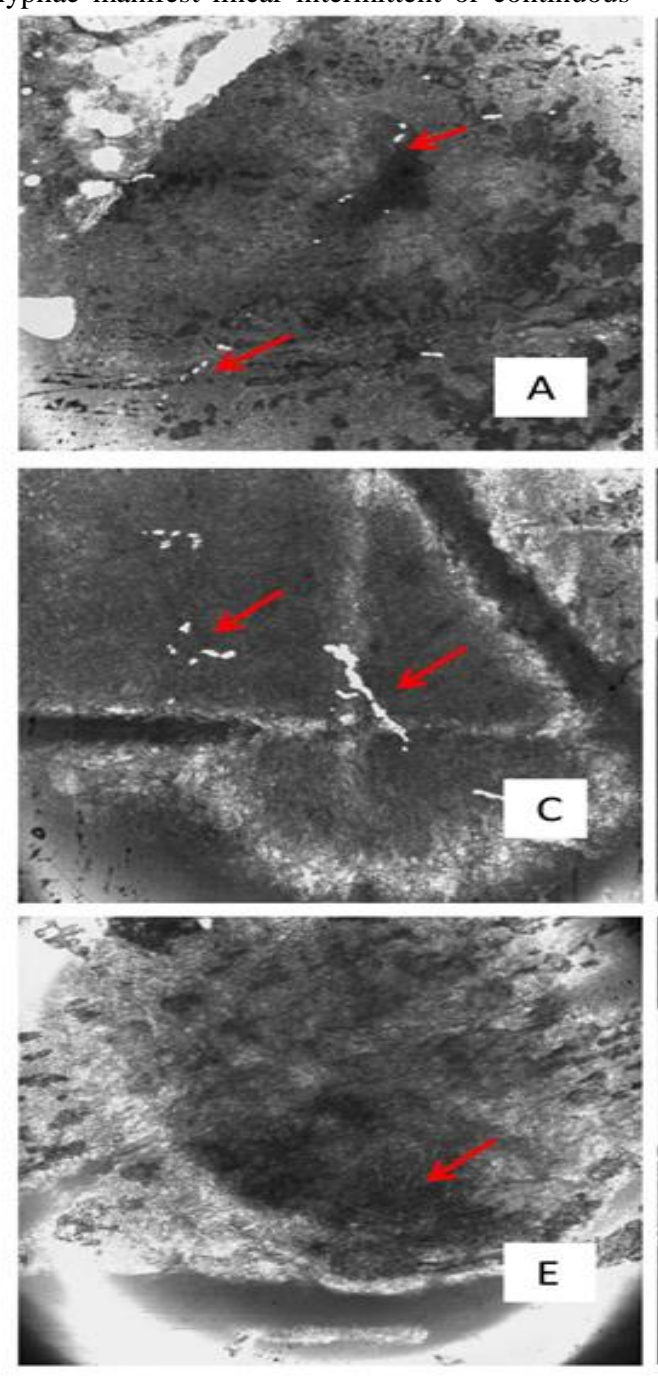

Figure3: The RCM images identification of hyphae from other substances A, C, and E show linear hyphal structure; B shows high refraction keratin; $D$ shows high refraction flake scales, and $F$ shows moderate refraction vellus hair structure

\section{Conclusions}

RCM could be independently applied to the diagnosis of tinea corporis and cruris. The positive rate of RCM diagnosis was slightly lower than that of fungal fluorescence microscopy. RCM seems to be improper to independently diagnose pityriasis versicolor. Fungal fluorescence microscopy in combination with RCM examination can significantly improve the positive rates of tinea manus and pedis.

\section{Acknowledgments}

Not applicable.

\section{Funding}

This work was supported by the National Natural Science Foundation of China (No.81903219).
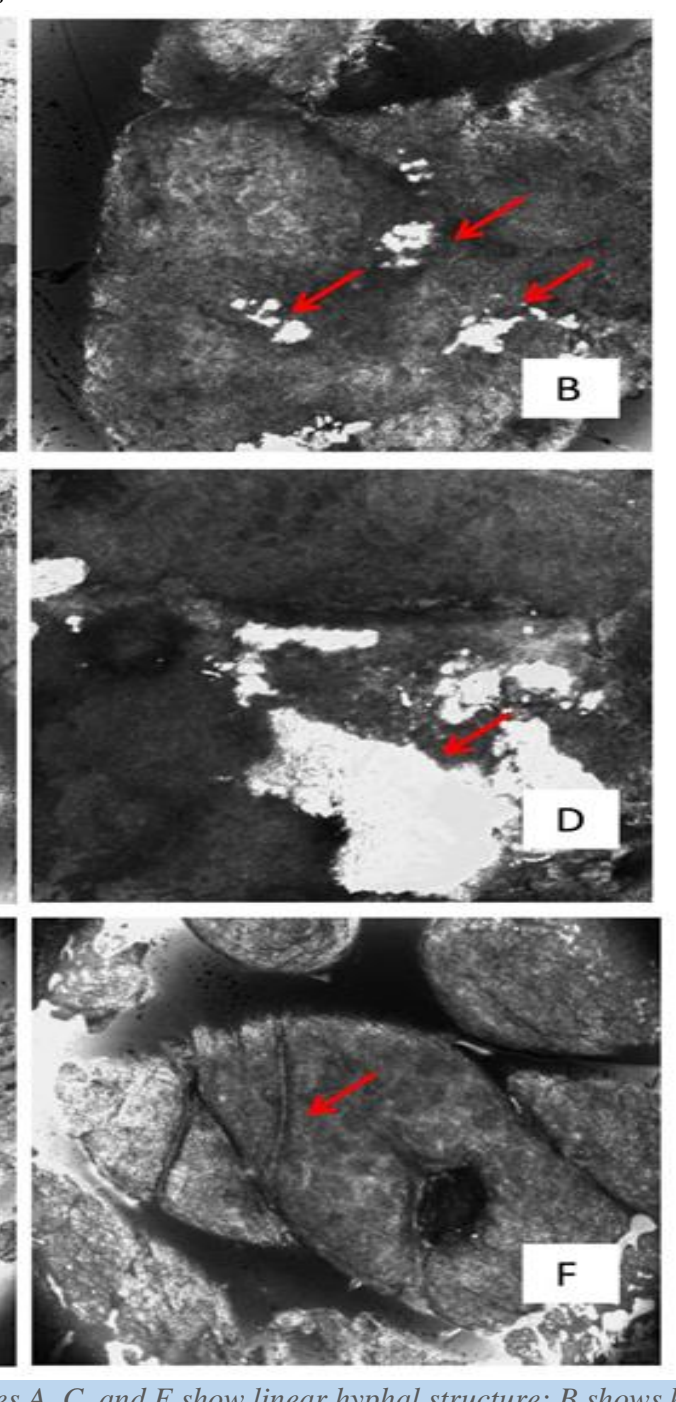

high refraction structure; while the scales formed irregular and larger area of homogeneous high refractive lamellar structures or some highrefractive keratinizing substances may also be regarded as hyphae mistakenly. Sometimes a small amount of slender and continuous medium refractive hyphae can be observed on the skin lesions of tinea versicolor patients. These hyphae are easily indistinguishable from the vellus structures on the skin surface (Figure3).

\section{Conflict of Interest}

The authors declare that they have no conflict of interest regarding this manuscript.

\section{References}

1. M C, et al. (2018). Epidemiology of fungal infections in China. Frontiers of medicine. 12(1): 58-75.

2. M A. (2010). Epidemiology of superficial fungal infections. Clinics in dermatology. 28(2): 197-201.

3. RR A, W TC. (2013). Dermatophytes. Current biology: CB. 23(13): 551-552.

4. SM Y, et al. (2010). Study to compare the efficacy and safety of fluconazole cream with flutrimazole cream in the treatment of superficial mycosis: a multicentre, randomised, double-blind, phase III trial. Mycoses. 53(6): 522-529. 
5. A W, B B. (2002). Topical treatment of common superficial tinea infections. American family physician. 65(10): 2095-2102.

6. Watanabe S. (2008). [Dermatomycosis--classification, etiology, pathogenesis, and treatment]. Nihon Rinsho. 66(12): 2285-2289.

7. Tampieri M.P. (2004). [Update on the diagnosis of dermatomycosis]. Parassitologia. 46(1-2): 183-186.

8. Serban E.D, et al. (2018). Role of In Vivo Reflectance Confocal Microscopy in the Analysis of Melanocytic Lesions. Acta Dermatovenerol Croat. 26(1): 64-67.

9. Andersen A.J.B, et al. (2018). In vivo characterization of pustules in Malassezia Folliculitis by reflectance confocal microscopy and optical coherence tomography. A case series study. Skin Res Technol. 24(4): 535-541.

10. Cinotti E, et al. (2015). Hair dermatophytosis diagnosed by reflectance confocal microscopy: six cases. J Eur Acad Dermatol Venereol. 29(11): 2257-2259.

11. Cinotti E, et al. (2014). Confocal microscopy for healthy and pathological nail. J Eur Acad Dermatol Venereol. 28(7): 853-858.

12. E C, et al. (2015). Reflectance confocal microscopy for mucosal diseases. Giornale italiano di dermatologia e venereologia: organo ufficiale, Societa italiana di dermatologia e sifilografia. 150(5): 585-593.

13. Chidambaram J.D, et al. (2016). Prospective Study of the Diagnostic Accuracy of the In Vivo Laser Scanning Confocal Microscope for Severe Microbial Keratitis. Ophthalmology. 123(11): 2285-2293.

14. Lupu M, et al. (2018). Non-invasive imaging of actinic cheilitis and squamous cell carcinoma of the lip. Mol Clin Oncol. 8(5): 640-646.

15. Tang Z, et al. (2020). Application and challenge of reflectance confocal microscopy in the diagnosis of pigmented nevus. Skin Res Technol.

16. Wurm E, et al. (2017). The value of reflectance confocal microscopy in diagnosis of flat pigmented facial lesions: a prospective study. J Eur Acad Dermatol Venereol. 31(8): 13491354.
17. Cinotti E, J.L Perrot and P. Rubegni. (2020). Beware of reflectance confocal microscopy artifacts when searching hyphae in acral skin. An Bras Dermatol. 95(1): 129-130.

18. Hui D, S Xue-cheng and X Ai-e. (2013). Evaluation of reflectance confocal microscopy in dermatophytosis. Mycoses. 56(2): 130133.

19. Ovrén E, et al. (2016). Dermatophytosis: fluorostaining enhances speed and sensitivity in direct microscopy of skin, nail and hair specimens from dermatology outpatients. Mycoses. 59(7): 436441.

20. Estela Cubells, J.R, et al. (2016). Fluorescence Microscopy as a Diagnostic Tool for Dermatophytosis. Am J Dermatopathol. 38(3): 208-210.

21. Mochizuki T, et al. (2020). Guidelines for the management of dermatomycosis (2019). J Dermatol. 47(12): 1343-1373.

22. SK Q, et al. (2015). Through the looking glass: Basics and principles of reflectance confocal microscopy. Journal of the American Academy of Dermatology. 73(2): 276-284.

23. Perrot J.L, et al. (2019). [Confocal microscopic examination of cutaneous mycosis is not restricted to research laboratories]. Ann Dermatol Venereol. 146(5): 385-386.

24. D F, F PC, and G M. (2015). Reflectance confocal microscopy: an effective diagnostic tool for dermatophytic infections. Cutis. 95(2): 93-97.

25. Cinotti E, et al. (2016). Reflectance confocal microscopy for cutaneous infections and infestations. J Eur Acad Dermatol Venereol. 30(5): 754-763.

26. Markus R, et al. (2001). A better potassium hydroxide preparation? In vivo diagnosis of tinea with confocal microscopy. Arch Dermatol. 137(8): 1076-1078.

27. D H, X-c. S, and A-e. X. (2013). Evaluation of reflectance confocal microscopy in dermatophytosis. Mycoses. 56(2): 130133.

28. T K and C AH. (2019). Superficial fungal infections. Australian journal of general practice. 48(10): 706-711.

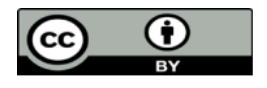

This work is licensed under Creative Commons Attribution 4.0 License

\section{To Submit Your Article Click Here: Submit Manuscript}

DOI: $10.31579 / 2578-8949 / 085$
Ready to submit your research? Choose Auctores and benefit from:

fast, convenient online submission

$>$ rigorous peer review by experienced research in your field

$>$ rapid publication on acceptance

$>$ authors retain copyrights

$>$ unique DOI for all articles

> immediate, unrestricted online access

At Auctores, research is always in progress.

Learn more www.auctoresonline.org/journals/dermatology-anddermatitiss- 